FORMATION Formation emploi

Revue française de sciences sociales

99 | juillet-septembre 2007

Les usages sociaux de la compétence

\title{
Faire valoir ses compétences : les pigistes et le placement de sujet
}

Asserting their competences: Free-lance journalists and how to sell their wares Die eigenen Fähigkeiten ins rechte Licht rücken: Freie Journalisten bieten ihre

Dienstleistung den Medien an

\section{Olivier Pilmis}

\section{QpenEdition}

Journals

\section{Édition électronique}

URL : http://journals.openedition.org/formationemploi/1480

DOI : 10.4000/formationemploi.1480

ISSN : 2107-0946

\section{Éditeur}

La Documentation française

\section{Édition imprimée}

Date de publication : 1 juillet 2007

Pagination : 75-86

ISSN : 0759-6340

\section{Référence électronique}

Olivier Pilmis, «Faire valoir ses compétences : les pigistes et le placement de sujet », Formation emploi [En ligne], 99 | juillet-septembre 2007, mis en ligne le 30 septembre 2009, consulté le 30 octobre 2020. URL : http://journals.openedition.org/formationemploi/1480 ; DOI : https://doi.org/10.4000/ formationemploi. 1480 


\section{DOSSIER}

\section{Faire valoir ses compétences: les pigistes et le placement de sujet}

Par Olivier Pilmis*

Le développement de la concurrence entre journalistes pigistes transforme les relations avec leurs employeurs. Le pigiste doit faire valoir ses compétences souvent avant même d'avoir l'occasion d'en faire la démonstration. Comment le pigiste parvient-il alors à orienter vers lui le jugement des employeurs?

$\mathrm{Au}$ cours des dernières décennies, la profession de journaliste s'est profondément modifiée. D'une part, le modèle économique sur lequel reposaient les entreprises de presse a été ébranlé par les concentrations entre groupes de presse et le développement des journaux gratuits (Le Floch et Sonnac, 2005). D'autre part, les pratiques de gestion de la main-d'œuvre journalistique se sont largement transformées ${ }^{1}$. Celles-ci reposent désormais davantage sur la sollicitation de pigistes extérieurs, les effectifs permanents diminuant relativement dans le même temps. La pige se caractérise par un paiement à l'article en fonction, généralement, dans la presse écrite, du nombre de signes. Les coûts de main-d'œuvre se voient alors réduits à la faveur d'une gestion par externalisation. Ainsi, en quarante ans, les effectifs des pigistes ont décuplé et leur part au sein de la population des journalistes a triplé.

Comme c'est le cas pour d'autres secteurs de l'économie, l'augmentation du nombre de pigistes est généralement considérée comme le principal

${ }^{1}$ L'explicitation du lien, complexe, entre ces deux évolutions, dépasse largement le cadre de ce texte. symptôme d'une précarisation du métier et de la situation des individus qui l'exercent (Devillard, 2002). Surtout, cet accroissement implique d'importantes transformations des conditions d'exercice du journalisme, voire de la conception même que les

Olivier Pilmis est ancien élève de l'IEP (Institut d'études politiques) de Paris, agrégé de sciences économiques et sociales, doctorant au Centre de sociologie du travail et des arts (EHESS) en thèse de sociologie sous la direction de P.M. Menger. Ses recherches portent sur les liens d'emploi sur des marchés du travail désintégrés, à travers l'exemple des journalistes pigistes et des comédiens intermittents. Elles se situent à l'intersection de la sociologie du travail, de la sociologie des professions et de la sociologie économique. Cet article reprend des résultats déjà présentés dans le cadre de la journée d'études "Les usages sociaux de la notion de compétence ", Paris, Conservatoire national des arts et métiers, mars 2006.

II a récemment publié : "Des "employeurs multiples" au "noyau dur" d'employeurs : relations d'emploi et concurrence sur le marché des comédiens intermittents », Sociologie du Travail 49 (3), 2007. 


\section{Encadré 1}

Les pigistes dans la population totale des journalistes titulaires de la carte

\begin{tabular}{|c|c|c|c|c|c|c|c|c|c|}
\cline { 2 - 10 } \multicolumn{1}{c|}{} & 1965 & 1970 & 1975 & 1980 & 1985 & 1990 & 1995 & 2000 & 2005 \\
\hline Effectifs & 710 & 950 & 1160 & 1600 & 2650 & 3920 & 4840 & 5890 & 6890 \\
\hline$\%$ & 7,1 & 7,9 & 8,5 & 9,6 & 12,1 & 14,7 & 17 & 18 & 19,7 \\
\hline
\end{tabular}

Source : CCIJP (Commission de la carte d'identité de journaliste professionnel)/IFP (Institut français de presse).

Les données de la CCIIP doivent être considérées avec prudence, pour au moins deux raisons. D'abord, ces données opposent les journalistes pigistes à des " journalistes mensualisés » en CDD (contrat à durée déterminée) et en CDI (contrat à durée indéterminée). Ce faisant, ces « journalistes mensualisés » comptent certains pigistes dans leurs rangs (Accardo, 1998). Ensuite, et surtout, ces données ne concernent que les détenteurs de la carte de presse, et sous-estiment de ce fait les pigistes. En effet, en raison des conditions d'exercice de leur activité, ils éprouvent davantage de difficultés à remplir certaines des conditions d'attribution de la carte de presse (en particulier, l'importance des revenus issus du journalisme). Ces chiffres ne sont donc qu'un indicateur d'une nette croissance de la population de pigistes.

individus peuvent avoir de la profession journalistique. Dans une certaine mesure, l'importance croissante de la pige participe du développement d'une nouvelle figure de journaliste, d'un nouveau mode d'exercice de la profession journalistique ${ }^{2}$.

À ce titre, le monde de la presse connaît des mutations similaires à d'autres pans de l'économie : flexibilisation, développement de formes atypiques d'emploi, externalisation, sous-traitance... L'étude du cas des pigistes est de ce fait susceptible de fournir des enseignements sur les effets de certaines des évolutions récentes du marché du travail. Notamment, la situation des pigistes se caractérise par la discontinuité de la relation d'emploi, liée à une moindre intégration des individus dans des organisations, en raison d'une organisation du travail par projet (Becker, 1988; Boltanski, Chiapello, 1999). L'individu n'est plus alors en contact avec son employeur que pendant la durée, plus ou moins éphémère, nécessaire à la réalisation d'une tâche particulière qui lui a été confiée. Dans le cas présent, la relation entre un pigiste et son employeur peut ne pas excéder le temps requis pour la fabrication (documentation, enquête, rédaction...) d'un article. In fine, l'activité journalistique ne se conçoit pas de la même manière selon que l'on est ou non pigiste. Ceux-ci opèrent désormais moins au sein

${ }^{2}$ Ces phénomènes rappellent ceux étudiés par P.-M. Menger (2002) à partir de l'exemple des artistes. d'organisations que sur un marché à l'entrée duquel chacun peut se présenter. Et tandis que les journalistes non pigistes se trouvent dans une situation proche de la mise à disposition de la force de travail typique de la relation salariée, la nature des liens entretenus par les pigistes avec les entreprises de presse se brouille. La limite entre relation d'emploi et relation commerciale s'avère ténue dans leur cas.

L'obtention d'un volume d'activité important et régulier suppose alors la mise en ouvre de techniques concurrentielles spécifiques, exprimées dans le «placement de sujet». Ce faisant, la palette des compétences requises par l'exercice «pigiste» de l'activité journalistique se trouve élargie; placer un sujet suppose en effet un certain nombre d'opérations. Celles-ci portent aussi bien sur l'objet même de la transaction, le futur article - qui doit être original et pointu et qu'il faut vendre - que sur la sélection d'un partenaire d'échange, l'entreprise de presse susceptible d'accepter « tel» article.

\section{LES COMPÉTENCES SUR LE MARCHÉ DE LA PIGE}

Le système même du travail à la pige s'apparente à un fonctionnement marchand plus qu'organisationnel, 
dans la mesure où le pigiste n'est que rarement intégré à une rédaction. Souvent, il n'est même pas physiquement présent dans les locaux. Grâce au téléphone ou à Internet, l'essentiel de son travail peut se réaliser sans que la moindre coprésence soit nécessaire. En revanche, sur le marché de la pige ${ }^{3}$, il se trouve régulièrement en concurrence avec l'ensemble des pigistes, pour des opportunités d'échange - en l'occurrence, des commandes d'articles de la part d'entreprises de presse, par ailleurs nombreuses. Le travail du pigiste est par nature incertain, d'autant plus que l'augmentation des effectifs de pigistes excède largement celle des entreprises de presse.

\section{Un marché ouvert}

La concurrence entre les pigistes est d'autant plus intense que le marché de la pige, bien qu'apparemment réglementé du fait de l'existence de la carte de presse, est un marché « ouvert $»^{4}$ (Paradeise, 1984) : l'accès n'y est pas conditionné à la détention d'une qualification. Il n'y a donc guère de certification durable, stable et irréversible de la qualité d'un pigiste. La formation suivie par l'individu peut alors être assez largement déconnectée des emplois qu'il obtiendra par la suite. De ce fait, les compétences du pigiste $^{5}$ ne peuvent être évaluées qu'au coup par coup, dans le cadre de relations d'emploi concrètes. Dans l'attente de ce verdict, l'opportunité d'emploi se présentera au pigiste jugé, en apparence, comme le plus compétent. Ce marché présente donc certaines caractéristiques de cercles vertueux/vicieux: seuls ceux dont on pense qu'ils sont compétents auront l'occasion d'en faire la démonstration. Être reconnu par un employeur comme compétent a priori est un atout majeur pour sortir victorieux de la concurrence opposant chaque pigiste à l'ensemble des autres.

\footnotetext{
${ }^{3}$ La conception du marché du travail ici mobilisée doit beaucoup aux travaux d'auteurs comme R. Swedberg (1998), P. François (2004) ou encore L. Karpik (1989).

${ }^{4}$ On dit d'un marché du travail qu'il est « ouvert » lorsque les travailleurs y exerçant ne sont protégés ni contre la concurrence externe (caractéristique du marché du travail en général) ni contre la concurrence interne (entre collègues).

${ }^{5}$ On entendra ici par "compétences» cet ensemble de «qualités », généralement présenté sous la forme du triptyque "savoir/savoir-faire/savoir être » (Reynaud, 2001) et qui suppose la valorisation, nouvelle par rapport à la logique plus ancienne de la "qualification », d'éléments tels que l'autonomie, la confiance, l'apprentissage, etc. (Paradeise, Lichtenberger, 2001).
}

Par la suite, cette incertitude relative aux compétences, ou à la qualité, d'un pigiste est susceptible d'être amoindrie. Une fois faite la démonstration de ses compétences, les relations entretenues par le pigiste avec les rédactions peuvent se répéter. Devenu un collaborateur régulier à la faveur des premières relations d'emploi, le pigiste voit s'accroître ses chances d'obtenir de nouvelles commandes émanant des rédactions auxquelles il est « abonné ». En témoigne en particulier le passage du démarchage des rédactions par le pigiste au rappel téléphonique de ce dernier par les rédactions. Le marché de la pige ne constitue pas en effet un marché purement atomistique, l'activité individuelle y est au contraire toujours susceptible d'être encastrée dans des réseaux de relations personnelles (Granovetter, 1985). Cet encastrement ${ }^{6}$ est à l'origine de la réputation et de la confiance accordées au pigiste (Lorenz, 1996 ; Chantelat, 2002). Celles-ci lui permettent alors de se prémunir, pour partie, de la concurrence marchande à laquelle il est autrement soumis, selon des mécanismes rappelant les mondes de l'art (Becker, 1988). Tous les pigistes ne sont donc pas égaux face à l'ouverture de leur marché du travail. L'inscription marchande du comportement du pigiste peut donc être dépassée dans certaines conditions : la concurrence à laquelle est soumise le pigiste peut se voir, à l'instar de l'incertitude sur sa qualité, diminuée. Cependant, cette diminution ne vaut pas suppression. L'activité du pigiste demeure soumise en permanence à une relative instabilité, puisque chaque individu voit ses compétences réévaluées (i. e. soumises à une nouvelle validation) à l'occasion de chaque nouvelle collaboration.

Mais confiance et réputation ne peuvent s'obtenir qu'au terme des premières relations avec une rédaction. Aussi l'obtention d'un jugement positif sur ses compétences est particulièrement impérative dans le cadre de collaborations nouvelles entre entreprise de presse et pigiste, avant même que celui-ci ait pu apporter la preuve de ses compétences et s'adapter aux conventions et routines en vigueur chez cet employeur. Ses compétences sont d'abord prêtées au pigiste avant d'être attestées par l'expérience.

\footnotetext{
${ }^{6} \mathrm{Il}$ permet également de rendre compte des bénéfices que peuvent tirer certains pigistes de l'usage de certains réseaux, sous la forme de conseils ou de recommandations.
} 
Orienter sur soi le jugement constitue donc une injonction particulièrement impérieuse dans le cas des pigistes les plus jeunes, débutants, et n'ayant que peu de ressources à faire valoir sur le marché (relations, expérience professionnelle, etc.). Pour autant, les pigistes plus aguerris ne sont pas à l'abri de ces difficultés. Les caractéristiques propres à ce marché du travail incitent en effet à distinguer l'ancienneté professionnelle de l'ancienneté d'une relation d'emploi particulière. La reconnaissance par un employeur de ses compétences est un problème que rencontrent tous les pigistes, quelle que soit leur ancienneté, dans le cas de relations d'emploi naissantes. Simplement, l'expérience professionnelle peut permettre de mieux y faire face.

\section{Bon journaliste et bon pigiste}

Sur ce marché du travail flexible, les compétences ne se trouvent pas cristallisées avec la même netteté que dans les autres entreprises et secteurs à l'organisation plus traditionnelle recourant à une logique de compétences (Tanguy, 1998). On ne peut guère en effet évoquer de postes dans le cas des pigistes, ni de taxinomies ou nomenclatures permettant d'évaluer, de manière formalisée, les compétences des individus. Les critères à l'aune desquels sont évaluées les compétences des pigistes se rapprochent donc ici davantage de ce que l'on pourrait décrire, en termes anthropologiques, comme des catégories de pensée indigènes permettant de faire la distinction entre les « bons » journalistes et les «mauvais ». Il apparaît alors qu'aux dispositions requises pour être reconnu comme un bon journaliste s'ajoutent celles nécessaires à la qualification comme bon pigiste.

Les compétences journalistiques comprennent en premier lieu celles nécessaires à l'exercice du métier de journaliste, celles dont tout journaliste doit faire la démonstration chaque jour dans l'exercice de sa profession. Elles englobent la maîtrise des modes journalistiques d'investigation, les diverses techniques d'écriture, le respect de la déontologie ${ }^{7}$, etc. Le champ de ces compétences s'élargit dans le cas de ce mode particulier d'exercice de l'activité journalis-

\footnotetext{
${ }^{7}$ Les travaux de C. Lemieux (2000) permettent d'en révéler certaines en creux, à travers l'étude des journalistes ayant commis des fautes.
}

tique qu'est la pige. Au noyau traditionnel des compétences journalistiques s'en ajoutent d'autres, dont la maîtrise est plus cruciale pour les pigistes que pour les journalistes permanents. Elles ne font généralement pas l'objet d'une formalisation dans le cadre d'enseignements au métier de journaliste 8 . Elles doivent alors être acquises «sur le tas» et constituent une dimension centrale de la socialisation professionnelle - sous la forme de «ficelles du métier » par exemple.

\section{Les compétences du pigiste au prisme du « placement de sujet»}

Une pratique propre au journaliste pigiste subsume l'ensemble de ses compétences: le placement de sujet. Cette pratique représente donc un point d'entrée pour l'étude de ces compétences caractéristiques de la pige. Son importance se comprend au regard de l'organisation du travail du pigiste. Salarié externalisé, travailleur à distance, le pigiste éprouve des difficultés à obtenir des commandes des employeurs avec lesquels il n'a pas noué de relations durables. De ce fait, au moins dans un premier temps, le pigiste est l'initiateur du lien avec l'employeur : il lui revient de le démarcher. Le terme de "placement de sujet» désigne ce pan particulier de l'activité de pigiste, qui contribue à le rapprocher de figures comme celle du travailleur indépendant ou du professionnel libéral.

Les compétences trouvent donc dans le placement de sujet une pratique à travers laquelle s'éprouver et s'apprendre : «tout comme l'on s'exerce à maîtriser les techniques de l'écriture journalistique, on peut apprendre à vendre un article à un rédacteur en chef $»^{9}$, assure ainsi un membre d'une association professionnelle de pigistes dans la revue publiée par l'association. Se faire embaucher par un employeur apparaît alors, à bien des égards, comme une dimension importante du travail lui-même. L'orientation sur soi du jugement est souvent un préalable indispensable à l'activité du pigiste : à ce titre, savoir

${ }^{8}$ Même si, signe de l'importance croissante de la population des pigistes, certaines écoles accueillent désormais des formations spécifiques à la pige.

${ }^{9}$ Christophe Belleuvre, «Épisode 1: Pigiste? un bon commercial!", Pigiste, 2, novembre 2004, p. 5. Cet article s'inscrit dans le cadre d'un dossier thématique intitulé « Vendre un papier $»$. 


\section{Encadré 2 \\ Méthodologie de l'enquête}

Ce texte s'appuie sur l'analyse d'entretiens semi-directifs auprès d'une trentaine de journalistes pigistes, réalisés au cours des années 2005 et 2006 . Ces entretiens portaient principalement sur les conditions d'exercice du métier de journaliste et sur le rapport de cette population au travail. La méthode employée pour constituer cet échantillon léchantillonnage en boule de neige, qui consiste à faire construire l'ensemble de l'échantillon d'études par les premiers individus rencontrés: amis, collègues, anciens condisciples, etc.) (cf. Jeffri, Heckathorn, 2000) pose problème quant à sa représentativité ; pour autant, elle demeure particulièrement adaptée à l'étude de populations professionnelles d'effectif restreint à forte interconnaissance. De plus, la recherche d'un échantillon représentatif se heurte au manque de données fines sur les caractéristiques sociodémographiques des pigistes.

L'échantillon ici analysé est assez largement féminin (environ deux tiers de femmes) et très majoritairement parisien, deux pigistes interrogés seulement habitant en dehors de la région parisienne (*). Quant à l'ancienneté professionnelle des membres de l'échantillon, elle varie en fonction de leur âge, certains n'étant pigistes que depuis quelques mois au moment de leur entretien, d'autres l'étant depuis plus de quinze ans. Toutefois, les « jeunes » pigistes prédominent dans l'échantillon ainsi constitué ll'âge moyen est de 33 ans). La presse écrite est largement présente, même si cet échantillon comporte quatre pigistes exerçant leur activité dans le secteur audiovisuel public. Leurs spécialités professionnelles diffèrent, bien que certains secteurs se distinguent légèrement, comme les presses culturelle, économique, féminine ou encore scientifique.

Les données ainsi collectées ont été complétées par le recueil de documentation de nature syndicale ou professionnelle, ainsi que par des observations menées auprès d'une association professionnelle de pigistes. On trouvera notamment, dans la suite de ce texte, des extraits d'articles publiés dans la revue d'une association professionnelle de pigistes, qui fournissent un éclairage intéressant sur la manière dont des pigistes " engagés » dans la défense de ce mode particulier d'exercice du journalisme en conçoivent les caractéristiques et contraintes.

(*) : Cependant, les technologies de l'information et de la communication permettent de s'affranchir de certaines distances et n'empêchent plus le pigiste de travailler avec des entreprises situées loin de son lieu de résidence.

placer un sujet devient presque aussi important que d'être capable de l'écrire.

Question: «Si Informatique Magazine ${ }^{10}$ s'arrêtait, comment vous feriez alors dans ce cas-là pour retrouver... Vous allez plutôt proposer des papiers à des rédactions sans les connaître ou...? »

Réponse : «En fait, ça, c'est un travail qu'on est censé faire tout le temps. Que le canard ferme ou qu'il ne ferme pas, on est censé (et c'est pas facile) on est censé partir du principe que demain tout peut s'arrêter. Donc que par conséquent, il est vital de continuer à prospecter, quoi qu'il arrive. Seulement, quand on bosse pour quatre canards en parallèle (ce qui n'est pas mon cas en ce moment) quand on bosse

${ }^{10}$ Tous les noms propres (individus comme publications) ont ici été modifiés. pour quatre canards en parallèle, bien évidemment, on a de moins en moins de temps pour prospecter, ce qui prend énormément de temps : prospecter, ça prend beaucoup de temps, parce qu'il faut regarder un petit peu à droite, à gauche, quels sont les titres qui peuvent correspondre, quels sont les sujets qui peuvent les intéresser. Est-ce que je connais quelqu'un? Est-ce que je connais pas quelqu'un? »

(Homme, 45 ans, presse informatique, 20 ans d'ancienneté ${ }^{11}$ )

\footnotetext{
${ }^{11}$ Il s'agit ici de l'ancienneté professionnelle, et non de l'ancienneté de l'une ou l'autre des relations d'emploi. Celle-ci ne peut être toujours connue avec précision : la remémoration de dates exactes d'entrée étant complexe en raison des parcours parfois chaotiques sur ce marché du travail (pour n'en prendre qu'un exemple: les premiers articles sont souvent rédigés durant, voire avant les études de journalisme).
} 
« C'était un journal que j'aimais bien lire, et c'est un ami qui m'a dit: "ben tiens appelle le rédacteur en chef de ma part, et vois si tu peux placer un papier". Et puis j'ai placé un papier, et puis deux, et puis trois, et puis voilà, quoi.»»

(Femme, 27 ans, presse magazine généraliste, radio, 9 ans d'ancienneté)

Le placement de sujet constitue donc une pratique caractéristique de l'inscription du pigiste sur un marché. En se centrant sur l'offreur de travail (ici, le pigiste), l'étude de ces compétences procède selon deux niveaux. D'une part, les compétences requises dans le contexte d'un placement de sujet portent sur l'objet même de la transaction, i. e. le sujet (une idée de reportage ou d'article). Il peut alors s'agir aussi bien de la nature même du sujet que des différentes manières de le présenter à l'employeur visé. D'autre part, ces compétences concernent l'identification d'un partenaire privilégié (une entreprise de presse) avec lequel procéder à un échange. Elles reposent alors sur une connaissance, souvent fine, du champ journalistique dans son ensemble.

\section{LES OPÉRATIONS SUR LE SUJET À PLACER}

S'interroger sur les modalités du placement suppose tout d'abord prêter attention à la nature de ce qui doit être placé. Le sujet, en tant qu'il doit être vendu, rassemble un certain nombre de caractéristiques qui sont le résultat d'autant d'opérations spécifiques de la part du pigiste. Placer un sujet suppose de parvenir à le vendre en en présentant les divers aspects (thème, mais aussi angle, résultats attendus, etc.), et rend de ce fait nécessaire son originalité, ce qui incite le pigiste à se spécialiser.

\section{Vendre un sujet}

Le placement de sujet requiert d'abord, de la part du pigiste, la mise en œuvre de compétences similaires à celles d'un entrepreneur. Le placement de sujet correspond à la situation où le pigiste présente un sujet «clés en main» à un employeur. Le pigiste compétent est alors celui qui parvient le mieux à vendre son sujet, c'est-à-dire à le présenter à l'employeur de la manière la plus attractive possible. Un premier groupe de compétences porte alors sur l'objet même de la transaction: le futur article. Il s'agit de montrer la qualité du futur produit et d'en faire une description assez détaillée pour que l'interlocuteur ait une claire perception de ce que sera ce produit final ${ }^{12}$. En effet, le terme indigène de « sujet» recouvre différentes dimensions : le thème général d'une investigation journalistique, une manière de l'aborder et de le traiter (l'angle), le mode d'investigation employé (la méthode), voire les résultats escomptés de l'enquête (ce qu'il va donc s'agir de montrer). Le placement de sujet ne requiert donc pas uniquement de présenter à l'employeur une simple idée susceptible de donner lieu à un article, mais de lui fournir l'ensemble des éléments permettant de savoir, par avance, ce que sera cet article une fois achevé. La teneur exacte du futur sujet fait souvent l'objet d'une négociation entre l'employeur et le pigiste afin de l'ajuster aux attentes des deux parties; toutefois, le travail du pigiste repose sur cette activité de sollicitation des employeurs autour de projets ${ }^{13}$. C'est à lui qu'il revient d'initier la future transaction afin de l'ajuster aux attentes des deux parties.

C'est dans cette optique qu'un pigiste envisage les formations qu'il anime :

«Ce qu'on fait, nous, dans les formations, c'est inciter les gens à avoir de l'imagination. L'exercicetype que je demande, quand je fais des formations et que les autres le font, c'est... de proposer des sujets bidons... Je vais demander aux stagiaires qui sont là d'inventer des angles, et de me mettre des canards en face à qui ils vont aller vendre les sujets. »

Décrivant la démarche nécessaire pour que les sujets proposés soient acceptés, ce même pigiste remarque alors qu'il est de nécessaire de «bien connaître le

${ }^{12}$ Le financement possible de la réalisation du sujet par l'employeur auprès duquel il a été placé vient confirmer cette analogie.

${ }^{13} \mathrm{La}$ démarche du pigiste vis-à-vis d'un employeur potentiel n'est pas sans évoquer, de ce point de vue, celle décrite par Pierre François à propos des ensembles de musique ancienne (François, 2004) : la négociation entre offreur et demandeur (qu'il s'agisse d'un sujet ou d'un concert de musique ancienne) ne porte pas tant sur le prix du produit que sur la définition de ses caractéristiques. 
journal, bien connaître l'actualité, trouver les angles qui vont avec, pour pouvoir placer les sujets ».

(Homme, 37 ans, presse agricole, président d'une association professionnelle de pigistes, 15 ans d'ancienneté)

Les termes employés par certains pigistes sont révélateurs de la pluralité des aspects à évoquer dans le cadre du placement de sujet. Ceux-ci emploient en effet, pour décrire le placement de sujets, des termes comme «synopsis», «scénario» ou encore « histoire ». Le sujet est présenté à l'employeur sous une forme narrative et globale. Cette impression est confirmée par la définition donnée par une pigiste dans les pages de la revue d'une association professionnelle : "Un synops c'est une vingtaine de lignes, à peine, qui définissent le sujet: l'actu, l'angle, la taille, un ou deux exemples concrets, les encadrés envisagés et, si possible, les illustrations. Le tout: dévoiler un peu l'idée mais pas trop...» (Barrais, 2004). Les compétences communicationnelles mobilisées dans le cadre du placement d'un sujet reposent largement sur des techniques qui peuvent être qualifiées de publicitaires. Le pigiste doit vendre (le verbe revient comme un leitmotiv dans les entretiens) un produit intellectuel encore inexistant et donc susciter une demande de la part du rédacteur en chef ou du chef de rubrique. Ces compétences communicationnelles permettent alors de vaincre la concurrence. Si bien que, pour ses pairs, « il n'y a pas à tergiverser, un bon pigiste est aussi un bon vendeur, un bon communiquant!» (Belleuvre, loc. cit.).

Question: "Comment tu t'y prends pour trouver des piges? Imagine, si demain Télévision Publique te...»

Réponse : "Alors là, je suis très mal. »

Question : «À ce point-là? »

Réponse : «Ah ouais, je suis très mal. Ca m'embêterait beaucoup parce que depuis 2002, ça va faire trois ans, je travaille qu'avec eux, donc tous mes autres contacts... sont plus qu'en jachère, donc il faut renouer les fils, reprendre des contacts, se mettre au courant de qui va faire quoi, qui aura besoin de qui. [...] Faut passer des coups de fil, faut aller voir des gens, il faut commencer à travailler sur des dossiers pour proposer des histoires, enfin c'est... tout un boulot, quoi. Qui est assez usant. »

(Homme, 35 ans, Télévision publique, 10 ans d'ancienneté)

Cette présentation du sujet ne correspond cependant pas à un pur dévoilement. Bien que détaillée, elle ne doit pas être trop complète, afin de ne pas encourir le risque de « vol » du sujet. En effet, après avoir refusé le sujet d'un pigiste, un employeur peut le confier à un journaliste non pigiste. Si les compétences sont des atouts concurrentiels qui favorisent la saisie d'opportunités de travail, les compétences communicationnelles dont doit faire preuve le pigiste pour placer un sujet reposent alors sur un équilibre entre divulgation et réserve.

Tel pigiste évoquera ainsi la «paranoïa» liée à la crainte de se faire voler ses idées par des concurrents pigistes : «donc, tu parles à personne, et surtout pas à tes confrères journalistes pigistes, parce que tout ce que tu fais, tu es susceptible de te le faire piquer [...] Y a pas de collègues. Que des concurrents. »

(Homme, 37 ans, presse agricole, président d'une association professionnelle de pigistes, 15 ans d'ancienneté)

La manière dont le pigiste présente et vend le sujet a un impact notable sur la réussite du placement luimême. Pour cela, il doit recourir à des techniques évoquant davantage des méthodes entrepreneuriales - il ne s'agit pas ici de vendre une simple idée de reportage mais bien un ensemble englobant l'idée et la manière dont elle sera concrétisée - visant à favoriser l'expression d'une demande pour le produit que le pigiste s'apprête à offrir. Ces techniques offrent une illustration des compétences dont doit savoir faire preuve le pigiste dans le cadre de son activité, bien qu'elles n'aient stricto sensu que peu de rapport avec le journalisme.

\section{Être original}

Cependant, l'importance, avérée ou seulement potentielle, de ces vols de sujet signale une autre dimension des compétences liées à l'objet même des transactions. En effet, les vols de sujet soulignent la nature de propriété intellectuelle du sujet, aux yeux des pigistes. À ce titre, le sujet à placer doit répondre à un critère 
essentiel: être original. Cette caractéristique du produit se transférant au producteur, l'originalité devient un trait nécessaire du « bon » pigiste. À l'autonomie dans l'exercice de son activité répond, comme en écho, l'originalité des produits en résultant.

«Mais en tout cas, j'ai pas de piston et j'ai pas de réseau donc j'ai pas d'autre choix, soit d'avoir un sujet ultra-original et d'être ultra-convaincante et assez... comment dire?... C'est un jonglage. C'est un jonglage entre une certaine assurance et en même temps une très grande souplesse et une très grande ouverture. Il faut pas du tout être en position de demande: il faut téléphoner, moi je téléphone au rédac en chef, c'est relativement d'égal à égal... »

(Femme, 38 ans, presse quotidienne régionale, 10 ans d'ancienneté)

L'originalité, souvent présentée comme le résultat de prédispositions personnelles ou de qualités individuelles, est une compétence, en tant qu'elle participe à l'évaluation de la qualité du pigiste par ses pairs et partenaires d'échange. Elle se révèle également importante dans la concurrence qui oppose le pigiste aux autres journalistes, qu'ils soient ou non pigistes. Compte tenu du nombre d'acteurs présents sur le marché de la pige, l'originalité permet de se démarquer de ses concurrents en proposant un sujet unique qui se distinguera aussi bien de ceux proposés par les autres pigistes que de ceux déjà commandés par le rédacteur en chef ou le chef de rubrique aux journalistes dont il a la charge. Originalité devient alors synonyme de plus-value, y compris dans les discours que tiennent les pigistes. De ce point de vue, l'originalité permet au pigiste de créer les conditions d'une transformation de la concurrence à laquelle il est initialement confronté en une concurrence de type monopolistique. Les produits journalistiques proposés par le pigiste ne sont alors qu'imparfaitement substituables à ceux des autres.

\section{Se spécialiser}

Cette modification de la structure même de la concurrence apparaît plus nette encore dans le cas de la spécialisation, qui va souvent de pair avec la recherche d'originalité. La spécialisation est perçue par les pigistes comme une nécessité sur le marché. Elle permet en effet d'investir un domaine sur lequel le pigiste est susceptible de développer à terme une quasi-expertise, i. e. d'avoir un apport important sous la forme de connaissances du thème que le généraliste ou le profane n'ont pas. Il peut ainsi se créer une niche d'activités le soustrayant à une partie de la concurrence à laquelle il se trouve confronté. Il n'est plus en concurrence avec l'ensemble des pigistes mais seulement avec ceux partageant une même spécialité. Cette spécialisation peut donc répondre à un investissement délibéré du pigiste ou n'être que le résultat d'une adaptation, presque mécanique, à une contrainte marchande. S'il est plus facile de placer un sujet pour les pigistes spécialisés que pour les généralistes, c'est d'abord et avant tout parce que cette spécialisation répond, de fait, à certaines évolutions du secteur de la presse. Tandis que le secteur de la presse d'informations générales (notamment quotidienne) tend à pâtir d'évolutions récentes (diminution $\mathrm{du}$ lectorat régulier, concurrence de supports comme les journaux gratuits ou encore Internet), la presse (magazine), spécialisée et/ou professionnelle, connaît un réel dynamisme (Le Floch et Sonnac, 2005). Dans ce cadre, la spécialisation peut devenir nécessaire pour qui souhaite réussir à obtenir des commandes de la part de magazines de ce type.

Question : «C'est une nécessité, la spécialisation?» Réponse: " C'est pas que c'est une nécessité, mais ça se fait comme ça, quoi. Enfin, "ça se fait comme ça”, ça s'est fait comme ça pour moi, parce que moi, je travaille régulièrement pour deux journaux et c'est les mêmes rubriques. Mais je sais pas s'il y a des pigistes, il y en a sans doute, qui font trois jours de la culture, le lendemain complètement autre chose. Je sais pas: c'est peut-être possible. Sans doute d'ailleurs, je sais pas. Mais je pense qu'après c'est plus difficile de vendre... ben de se vendre auprès des journaux, sauf si ça fait dix ans qu'on fait un peu de culture, un peu d'économie et un peu de sport, parce que là, on est multi-cartes... »

Question : «Mais quand vous dites se vendre auprès des journaux... »

Réponse : «Ben c'est comme quand on recherche un emploi dans n'importe quel autre domaine, faut quand même dire: "moi, je suis compétente dans tel truc" " (Femme, 30 ans, presse économique, 5 ans d'ancienneté). 
La spécialisation constitue ainsi une technique concurrentielle pour augmenter les chances de réussite d'un placement de sujet. Elle répond notamment à la structure du marché du travail des pigistes, et plus généralement de la presse, en mettant en évidence sa segmentation. Ce rôle joué par la spécialisation se trouve de ce fait à la charnière des deux types d'actions entre lesquelles le placement de sujet peut être décomposé. Le premier porte sur l'objet même de la transaction, le second sur l'entreprise de presse auprès de laquelle sera effectué ce placement.

\section{LA DÉSIGNATION DU PARTENAIRE D'ÉCHANGE IDÉAL}

En effet, la réussite d'un placement de sujet n'est pas conditionnée aux seuls éléments constituant le sujet lui-même. Elle dépend également de l'entreprise auprès de laquelle le placement est effectué. La désignation d'un partenaire d'échange est de ce fait aussi importante que ce qu'il va être question d'échanger. Trouver l'employeur avec lequel l'échange a le plus de chances de se réaliser est de ce fait central. Ceci suppose de repérer le titre auprès duquel la plusvalue que peut représenter le pigiste est la plus élevée, mais aussi, plus généralement, de développer une connaissance indigène fine du champ journalistique afin de se repérer sur les différents segments qui le composent.

\section{Trouver le bon titre}

En plaçant l'individu sur des segments particuliers du marché du travail, la spécialisation favorise la transaction avec un employeur. La plus-value qu'apporte le pigiste ne se conçoit en effet que relativement à un employeur : elle est maximale quand le sujet correspond à un domaine peu ou pas représenté au sein de la rédaction permanente, tout en s'inscrivant dans la ligne éditoriale du média employeur. L'originalité est une compétence d'autant plus efficace qu'elle est reliée à une certaine vision de l'employeur et des domaines de spécialité pour lesquels il a le plus de chances d'accepter un sujet venant d'un pigiste. Si les qualités intrinsèques du sujet ainsi que la manière de mettre en valeur impor- tent, ce premier type de compétences, portant sur l'objet de la transaction, ne saurait épuiser l'ensemble des compétences propres au pigiste. Le repérage de l'employeur avec lequel l'échange a le plus de chances d'aboutir est également une étape importante. De plus, il apparaît nécessaire de connaître le lieu où ces placements de sujet ont le plus de chances de réussir en fonction du type de spécialisation du pigiste.

«Lula, L'Éthique, c'est le quotidien, en gros. Et les autres c'est sur un sujet particulier. S'il y a un sujet à un moment que j'ai vraiment envie de défendre ou, euh, dans ce cas-là... je vais appeler d'autres personnes. Donc c'est vrai qu'il y a un an pile, bon, y a vraiment eu un gros truc du mouvement anti-pubs, moi, j'ai vraiment beaucoup fait partie de ce truc-là. Donc, dans ce cas-là, j'ai appelé, ben justement Kultur, Civis, etc. Parce que je sais que ça peut les intéresser, et parce que c'était vraiment un sujet que j'avais envie de défendre. Après, bon, le commerce équitable, je vais pas aller appeler Civis: déjà, parce qu'ils y sont, donc j'ai pas de valeur ajoutée à leur apporter... Oui, parce que en fait, le problème, il est là aussi, c'est que... qu'à un moment, c'est... les canards, ils ont leur structure, ils suivent l'actualité. Donc, c'est "est-ce que moi, en tant que pigiste, je peux vraiment leur apporter quelque chose?", auquel cas ils ont besoin de moi. Dans ce cas-là, je peux aller les voir en leur disant: "bon, ben là, j'ai un truc, ça va vous intéresser. $Y$ a pas de journaliste... enfin, c'est ma spécialité, je suis spécialiste de ça, quoi" [...] Dans ce cas-là, ça a un sens mais sinon, y a plein de choses ça a aucun sens que j'aille leur proposer. Ils vont me dire: "oui, mais nous, on le fait déjà en interne. On a déjà nos pigistes qui sont là-dessus" " .

(Femme, 28 ans, presse jeunesse et presse associative, 7 ans d'ancienneté)

Le lien entre spécialisation et caractéristiques de l'employeur est la première illustration de cet autre ensemble de compétences mobilisables par le pigiste dans le contexte d'un placement de sujet. Non seulement il importe de ne pas vendre n'importe quel sujet, et il faut que la plus-value que représente le pigiste soit aussi élevée que possible, mais cette plus-value est d'autant plus importante que l'employeur sera choisi avec soin. Ce choix suppose la mobilisation de 
connaissances sur l'entreprise de presse elle-même, ainsi qu'une relative familiarisation avec son mode de fonctionnement. En particulier, une compréhension de la façon dont le travail s'organise et se divise au sein de la rédaction facilite le succès du placement de sujet : l'espace dont disposera le pigiste pour placer un sujet sur un thème particulier sera d'autant plus large qu'aucun journaliste, au sein de la rédaction, n'en est spécialiste.

\section{Se repérer} dans le champ journalistique

La détermination d'un partenaire d'échange requiert cependant d'autres connaissances que la seule identification d'un titre et d'une spécialité professionnelle. Le placement de sujet nécessite la mobilisation d'autres types de savoirs, plus réflexifs, ayant trait à la connaissance du champ journalistique. Ceux-ci constituent en effet autant de repères pour situer l'ensemble des acteurs les uns par rapport aux autres, et donc mieux appréhender les différences, minimes mais néanmoins réelles, qui peuvent se traduire par des variations importantes des chances de succès d'un placement de sujet.

Une productrice de Radio France, à propos de ses expériences en tant que pigiste :

«Et si je pose aussi des questions dans cet angle-là, ça va pouvoir coller, là, quoi. Des fois, j'ai vu des pièces de théâtre faits par des mecs issus de l'immigration à Strasbourg, j'ai pu le vendre à X. et à Y. Les deux étaient intéressés. Je le montais différemment, les formats n'étaient pas les mêmes, mais ils ont été vendus dans les deux. »

(Femme, 40 ans, productrice Radio France, 15 ans d'ancienneté)

«Je fais beaucoup de propositions parce que j'ai énormément d'infos, je sais ce qui les intéresse, je sais les sujets qui les préoccupent, parce que chaque rédaction est plus préoccupée que... sur certains sujets que d'autres. Parce que chaque rédaction a son lectorat, sa cible, son marketing, donc, euh, je sais bien qu'avec tel journal, on s'intéresse plus à tels dossiers, à tels sujets... Au Magazine d'info plus qu'à Enquête, plus qu'à L'hebdo, plus qu'au Chroniqueur, et chacun a son... sa rédaction si tu veux.
Donc, je sais qu'au Magazine d'info, tel sujet va les intéresser, tel sujet va pas les intéresser ou... Donc je leur propose et puis après on... Ils ont bien vu que la qualité de mes informations, des informations que je publiais chez eux amenait des reprises, donc ils ont $v u$ que c'était intéressant pour eux de publier certaines de mes informations. »

(Homme, 40 ans, magazines d'actualité, 15 ans d'ancienneté)

Ce dernier extrait d'entretien est particulièrement révélateur de cette connaissance fine du champ journalistique, assez répandue chez les pigistes. Elle rappelle à certains égards la sociologie du champ journalistique développée dans la lignée des travaux de Pierre Bourdieu (Bourdieu, 1996; Benson, 1999), sans qu'il soit possible de savoir si la vision développée ici par les pigistes est indigène ou s'il s'agit, au contraire, d'une application à leur propre cas d'une théorie avec laquelle ils sont souvent familiarisés, et qui servirait de grille interprétative des évolutions récentes. Cette connaissance du champ journalistique porte en premier lieu sur les caractéristiques générales des entreprises, e.g. presse masculine versus presse féminine, ou encore généraliste versus presse professionnelle ou spécialisée. Mais elle ne se limite pas à ces éléments opposant de manière assez grossière les différentes entreprises de presse, et comporte d'autres distinctions, au sein d'une même catégorie, en termes d'angle ou de lectorat notamment. Les pigistes développent alors une opposition entre médias occupant des segments relativement proches du marché de la presse. Dans cette optique, le placement de sujet consiste à s'adapter aux caractéristiques d'un marché très fortement segmenté, selon plusieurs dimensions : types de média (presse écrite, radio, télévision) aussi bien que publics visés ou thèmes abordés. C'est à partir de ces catégories de perception que le pigiste déterminera le média correspondant le mieux au sujet qu'il souhaite placer, et donc celui auprès duquel ce placement a le plus de chances de réussir. Un grand nombre d'éléments sont pris en compte afin de maximiser les chances de succès du placement de sujets. La sélection fine par le pigiste d'un employeur particulier est une condition importante pour que les opportunités d'emploi se présentent concrètement. 
«Moi, je travaille pour L'Éthique. L’Éthique, c'est un site Internet qui est financé par la Caisse des Dépôts et Consignations, qui s'occupe de développement durable, et qui est sur Internet et sinon je travaille pour Lula. C'est chez Balan Presses, donc, chez Millard Presses. »

Question: "Oui, j'ai cru comprendre que tout le monde rachète tout le monde... enfin il y a deux, trois groupes qui rachètent tous les autres.»

Réponse : "Ouais, t'es obligé de t'intéresser vraiment au... à la concentration de... enfin, de... des groupes de presse dans ces vingt dernières années parce que... ça a complètement bouleversé le métier.»

(Femme, 28 ans, presse jeunesse et presse associative, 7 ans d'ancienneté)

Outre les éléments susceptibles de situer les diverses entreprises de presse dans le champ journalistique, ces compétences reposent également sur une connaissance des caractéristiques réticulaires du segment de marché que le pigiste souhaite investir. Il peut alors s'agir de la connaissance des réseaux industriels liés à la concentration des groupes de presse, notamment en ce que ces phénomènes de concentration rendent possibles des comportements stratégiques (placer un sujet auprès d'un employeur $x$ pouvant être une étape avant de placer un sujet auprès d'un employeur $y$ appartenant au même groupe de presse $)^{14}$. Les compétences cognitives dont fait preuve le pigiste s'étendent aux réseaux participant à la structuration du champ journalistique.

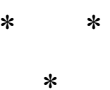

Les pigistes sont placés dans une situation d'incertitude corrélative à leur inscription dans la sphère marchande. Confrontés à une concurrence d'autant plus exacerbée que les barrières à l'entrée de ce marché sont minces, ils doivent maîtriser des techniques variées qui excèdent le seul cœur des compé-

${ }^{14}$ À l'inverse, cette concentration peut interdire au pigiste l'accès à des pans entiers du marché du travail, la rupture conflictuelle avec un titre d'un groupe de presse pouvant s'accompagner d'un arrêt de la collaboration avec l'ensemble des titres le composant. tences professionnelles, lequel regroupe notamment ce qui relève des normes d'investigation et d'écriture journalistiques ou encore les éléments constituant la déontologie professionnelle. Si ces compétences particulières s'imposent aux pigistes, parfois même avec davantage de vigueur (comme c'est le cas du respect des formats), elles ne suffisent pas, pour le regard indigène, à distinguer un « bon » pigiste d'un « mauvais ». L'exemple des pigistes illustre donc la manière dont certaines évolutions récentes des marchés du travail conduisent à des modifications de l'activité elle-même, ainsi que des rapports que les individus entretiennent avec elle. Le contenu du travail même de journaliste se trouve modifié à la faveur du développement de la pige. Cantonnés à l'extérieur de l'organisation et travaillant le plus souvent à distance, les pigistes adoptent certains traits du travailleur indépendant et de l'entrepreneur : la frontière entre relation d'emploi et relation commerciale se brouille dans le cas de ces travailleurs qui initient eux-mêmes la relation avec un employeur et participent, de manière déterminante, à la création et à la définition de leur propre emploi. Dans leur cas, le marché du travail est aussi, de manière souvent indissociable, un marché des produits : l'obtention d'un travail correspond à la vente d'un produit journalistique auprès d'une entreprise de presse. L'environnement concurrentiel dans lequel les pigistes évoluent et l'incertitude sur leur qualité professionnelle rendent impérative l'orientation sur soi du jugement des entreprises de presse. Cette injonction est à l'origine du développement de nouvelles compétences mobilisables dans le cadre de l'activité. Ce faisant, celle-ci se développe vers son amont : aux tâches de réalisation du produit journalistique s'ajoutent toujours davantage ${ }^{15}$ les tâches de conception, et de promotion, de ce produit.

\footnotetext{
${ }^{15} \mathrm{Il}$ serait en effet caricatural de ne prêter qu'aux seuls pigistes cette dimension du travail journalistique.
} 
Accardo A. (dir.) (1998), Journalistes précaires, Bordeaux, Le Mascaret.

Barrais D. (2004), «Vendre un papier? Un défi quotidien... », Pigiste, 2, novembre, p. 6.

Becker H.S. (1988) [1982], Les Mondes de l'art, Paris, Flammarion.

Benson R. (1999), "Field Theory in comparative Context : A new Paradigm for Media Studies", Theory and Society 28 (3), pp. 463-498.

Boltanski L., Chiapello E. (1999), Le Nouvel esprit du capitalisme, Paris, Gallimard.

Bourdieu P. (1996), Sur la télévision, suivi de L'emprise du journalisme, Paris, Liber.

Chantelat P. (2002), «La Nouvelle Sociologie Économique et le lien marchand: des relations personnelles à l'impersonnalité des relations ", Revue française de sociologie 43 (3), pp. 521-556.

Devillard V. (2002), « Les trajectoires des journalistes détenteurs de la carte de presse entre 1990 et 1998 : la montée de la précarité », Communication \& langages, $\mathrm{n}^{\circ} 133$, pp. 21-32.

François P. (2004), «Prototype, concurrence et marché: le marché des concerts de musique ancienne », Revue française de sociologie 45 (3), pp. 529-559.

Granovetter M.S. (1973), "The Strength of Weak Ties", American Journal of Sociology, 78 (6), pp. 1360-1380.

Granovetter M.S. (1985), "Economic Action and Social Structure: The Problem of Embeddedness", American Journal of Sociology 91 (3), pp. 481-510.

Heckathorn D., Jeffri J. (2000), "Finding the Beat: Using respondent-driven sampling to study jazz musicians", Poetics 28 (4), pp. 307-329.
Institut Français de Presse (Université PanthéonAssas Paris-II) (1991), Les journalistes français en 1990. Radiographie d'une profession. CCIJP/MCC Service Juridique et Technique de l'Information, La Documentation française.

Karpik L. (1989), "L'économie de la qualité », Revue française de sociologie 30 (2), pp. 187-210.

Le Floch P., Sonnac N. (2005) [1 1 éd. : 2000], Économie de la presse, Paris, La Découverte.

Lemieux C. (2000), Mauvaise presse. Une sociologie compréhensive $d u$ travail journalistique, Paris, Métailié.

Leteinturier C. (2001), « Les journalistes titulaires de la carte de presses en 1999. Etude sociodémographique d'après les données de la CCIJP au $1^{\text {er janvier }}$ 2000 », in Valérie Devillard et al., Les journalistes français à l'aube de l'an 2000. Profils et parcours. Paris, Éditions Panthéon-Assas, pp. 35-120.

Lorenz E. (1996), «Confiance, contrats et coopération économique », Sociologie du travail, 38 (4), pp. 487-508.

Menger P.-M. (2002), Portrait de l'artiste en travail. Métamorphoses du capitalisme, Paris, Seuil.

Paradeise C. (1984), «La marine marchande française : un marché du travail fermé ? », Revue française de sociologie 25 (3), pp. 352-375.

Paradeise C., Lichtenberger Y. (2001), « Compétence, compétences », Sociologie du travail, 43 (1), pp. 3348.

Reynaud J.-D. (2001), «Le management par les compétences : un essai d'analyse », Sociologie $d u$ travail, 43 (1), pp. 7-31.

Supiot A. (dir.), Le travail en perspectives, Paris, LGDJ, pp. 545-562. 
Swedberg R. (1998), Max Weber and the Idea of Economic Sociology, Princeton, Princeton University Press.
Tanguy L. (1998), « De l'évaluation des postes de travail à celle des qualités des travailleurs. Définitions et usages de la notion de compétences », in

\section{Résumé}

\section{Faire valoir ses compétences : les pigistes et le placement de sujet}

Olivier Pilmis

La hausse du nombre des pigistes parmi les journalistes suscite un changement des pratiques sur ce marché du travail. En effet, le pigiste est souvent en concurrence avec d'autres pigistes pour obtenir des opportunités d'emploi avec les différents employeurs. Le placement de sujet désigne alors l'ensemble des techniques mises en œuvre par le pigiste afin d'orienter la demande de l'entreprise vers son offre particulière de travail et lui vendre un sujet. Sa réussite requiert aussi bien des compétences propres à l'ensemble des journalistes que des compétences plus spécifiques, souvent acquises par la pratique.

\section{Mots-clés}

Journaliste, compétence, travail précaire, concurrence

Journal of Economic Literature : J 44, J 24 


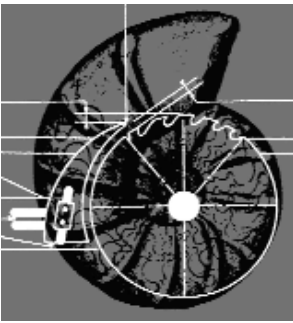

\section{TRAVAIL, GENRE ET SOCIÉTÉS}

I a $r$ e v u e d u M a ge

\section{$18 / 2007$}

NOVEMBRE 2007

PARCOURS

Entretien avec Viviane Isambert-Jamati "Le féminisme est, pour moi, une évidence" propos recueillis par Marlaine Cacouault-Bitaud et Rebecca Rogers

DOSSIER

Formation et orientation : I'empreinte du genre Nathalie Bosse et Christine Guégnard Clotilde Lemarchant Dominique Epiphane Françoise Vouillot

MUTATIONS

Claudio Zanier

CONTROVERSE

autour du thème

y a-t-il une féminisation de la vie politique? Françoise Gaspard, Eléonore Lépinard, Janine Mossuz-Lavau, Marion Paoletti, Mariette Sineau, Yves Sintomer

\section{CRITIOUES}

Directrice de la revue : Margaret Maruani

Secrétaire de rédaction : Anne Forssell

Comité de rédaction : Philippe Alonzo, Tania Angeloff, Marlaine CacouaultBitaud, Laura Lee Downs, Delphine Gardey, Annie Labourie-Racapé, Jacqueline Laufer, Thérèse Locoh, Monique Meron, Sophie Ponthieux, Isabelle Puech, Hyacinthe Ravet, Rachel Silvera, Françoise Vouillot.

Revue semestrielle publiée avec le concours du CNRS et du CNL.

Travail, genre et sociétés - CNRS - 59, rue Pouchet - 75017 Paris tél. 0140251037 - fax 0140251170 - e-mail tgs@tgs.cnrs.fr http: //www.tgs. chrs. fr

\section{Abonnements :}

Revues Armand Colin - Service Abonnements - 5, rue Laromiguière

75240 Paris cedex 05 - tel 33 (0) 140464989

mèl : infos@armand-colin.fr

http: / /www. armand-colin.com 